\title{
Relay System Model with Contact Bounce and Flexible Beam
}

\author{
Rui Gao $^{1}$ Ivar Torstensson ${ }^{2}$ \\ ${ }^{1}$ Modelon K.K. Roppongi 1-10-3-209, Minato ku, Tokyo 106-0032, Japan, \\ rui.gao@modelon. com \\ ${ }^{2}$ Modelon, AB, Sweden, Anders Carlssons Gata 14 SE-417 55 Göteborg, Sweden \\ ivar.torstensson@modelon.com
}

\begin{abstract}
In this paper, an electromagnetic relay's dynamic modeling problem is discussed. The moveable part of the relay is modeled as Euler-Bernoulli beam, while the impact phenomenon between the moveable and stationary poles is captured by $2 \mathrm{D}$ contact model using polygon to represent the contact pole profile. For the completeness of the system, the electromagnetic driving force is modeled using electrical and magnetic components from Modelica Standard Library. The simulation results show that the model reproduces the electromagnetic relay's physical behavior under normal operation condition. In addition, the model can be used to investigate the effect of abnormal scenario such as the contact surface profile change under material erosion. This application illustrates that the proposed method and model can be applied to the electromagnetic relay's simulation-based design as one-stop tool.
\end{abstract}

Keywords: electromagnetic relay, contact bounce, polygon, flexible beam

\section{Introduction}

The electromagnetic relay is one of the most widely used devices in industries and daily life. Electromagnetic relays provide a well proven solution to switching loads in a variety of applications. However, relays are known for their limited reliability due to mechanical wear of internal switching elements, essentially the life of the relay may be determined by the life of the contacts. The contact bounce phenomenon between the stationary and moveable contact poles has been studied from various perspectives. The research results show that it is the main reason for electric abrasion and material erosion of the contact poles, which affects the switching reliability.

Many studies have been focused on the characteristics of the contact bouncing mechanism through both experimental and analytical investigation, like the bounce time, first bounce amplitude, factors to affect contact bounce (McBride et al, 1992; Kondo et al, 2019);

In the dynamic modeling and simulation perspective, researchers are mainly concentrated on FEM method and tools. MATLAB/Simulink is used to model a simplified relay model represented by ordinary differential equation to capture the contact bounce (Nouri et al, 1997), indicating that lumped model is as effective as FEM in modeling relay system. Combined approach of distributed and lumped modeling is reported (Xiong et al, 2009), where finite difference method for reed system dynamic analysis with 1D spring mass system for the contact force calculation is studied. However, the friction force is not considered.

Modelica is a suitable language for multi-discipline modeling and simulation and has been widely used across industries. However, Modelica's traditional network model of component module encounters challenge in electromagnetic relay modeling. The difficulties stem from the innate features of relay where the moveable contact pole mounted on the flexible beam is driven by electromagnetic force, and reaction force from impact is discontinuously acted upon. Especially, the simulation performance under this specific method to capture discontinuous contact phenomenon has not yet been established with common acceptance.

There is a commercial library for flexible multibody modeling (Hackerman et al, 2006); Claytex developed another commercial library Flexbody. Both libraries need Finite Element model's results and importing them into Modelica environment after pre-processing. This dependency to the pre-processor makes the Modelica users difficult to use these solutions. A motorbike swingarm modeling and analysis application using Modelica multibody and parameters obtained through FEM package is reported (Gianni et al 2014), where authors try to alleviate the dependency to FEM software. On the other hand, an efficient Modelica modeling method that is independent with FEM code is investigated for flexible beam (Ericsson et al. 2015). We will use the model created based on this approach in the relay modeling in this paper.

Another difficulty in relay mechanical modeling is how to handle the discontinuous contact phenomenon. Modelica Standard Library (MSL) provides a 1D model WithStopAndFriction to capture the slip and stick, where hard stops are included using mode transition. This proves to be a practical method for simple 1D application. However, the collision occurred beyond the translation direction cannot be applied. An early effort to handle the contact modeling under multibody 
paradigm is reported where an external $\mathrm{C}$ package is used for collision detection (Otter et al. 2005). A free Ideal Contact Library is developed in Modelica multibody framework. It is easy to use but limited to the cases that the contact point location is predictable beforehand (Oestersötebier et al, 2014). A generic Modelica framework for multibody contacts and Discrete Element Method has been proposed (Elmqvist et al 2015). Component-based 3D modeling for dynamic systems inspired by game software is studied recently (Neumayr and Otter, 2018, 2019) where the limitation of the current Modelica specification is analyzed and a new way of $3 \mathrm{D}$ modeling technique implemented by Julia language with Modia environment is illustrated, e.g. Modia3D prototype.

Though the latest research results are quite promising to improve the multi-body system contact modeling with variable step solver, the deformation of flexible body has not been mentioned. To address electromagnetic relay's modeling issues like how to develop an efficient, light-weight package that covers flexible beam, contact bounce, and electromagnetic driving in order to realize simulation-based design is of wide interest to industries. This is the main focus of this paper.

The rest of the paper is organized as follows. Section 2 describes the relay system modeling. Section 3 illustrates the simulation results for normal case and abnormal case. A conclusion is summarized at the Section 4.

\section{Relay Modeling}

A relay is an electromagnetic switch used to switch high voltage or current using low power circuits. Its typical structure is shown in Figure 1, containing an electrical coil, magnetic core, yoke, armature, moveable and stationary contact.

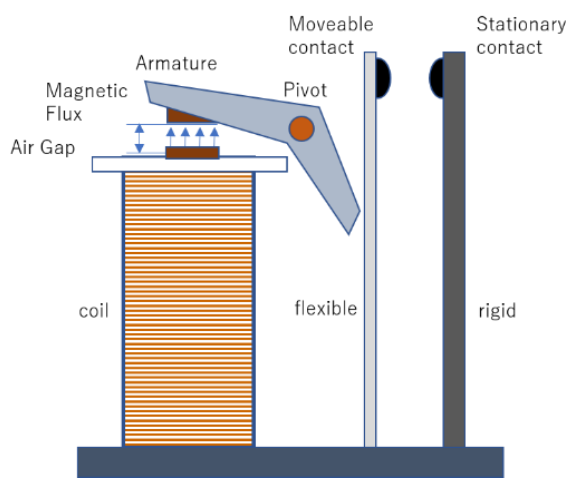

Figure 1. Typical electromagnetic relay structure.

An electromagnetic relay can always be separated into two subsystems (electromagnetic and mechanical) which interact with each other. The electromagnetic subsystem is governed by Maxwell's equations whereas the mechanical subsystem obeys Newton's law.

\subsection{Flexible beam}

The moveable beam in Figure 1 can be equivalently modeled as cantilever beam in Figure 2. This is one challenge to model continuum with Modelica without relying on FEM software, while the simulation performance is expected to be at 1D simulation tool level.

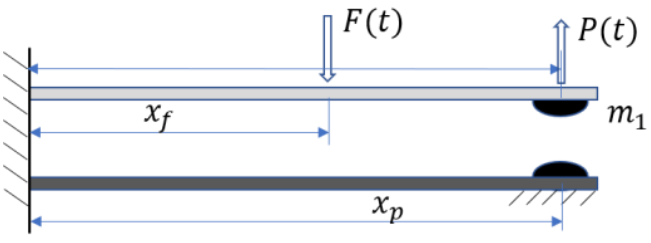

Figure 2. Mechanical part of the relay

Since the cross-section area of the beam is constant, the linear stress strain law can be applied to get motion equation as below (Xiong et al 2009).

$$
\begin{aligned}
& {\left[\rho A+m_{1} \delta\left(x-x_{p}\right)\right] \frac{\partial^{2} y}{\partial^{2} t}+E I(1+i g) \frac{\partial^{4} y}{\partial^{4} x}} \\
& =F(t) \delta\left(x-x_{f}\right)-P(t) \delta\left(x-x_{p}\right)
\end{aligned}
$$

where $E$ is the elastic modulus of the beam, $\rho$ the density, $A$ and $I$ are respectively the area and the moment of inertia of the beam cross-section, $g$ is the hysteretic damping ratio where $(1+\mathrm{i} g)$ is complex number, $\delta(x)$ Dirac function and $P(t)$ the contact force during the impact. Cantilever beam using Euler-Bernoulli theory is typical suitable to the beams for which the length is much larger than the thickness.

For the moveable beam in Figure 2, the ratio of length over thickness is over several dozens. Euler-Bernoulli beam implemented in Modelon Base Library (MBL) is used in the successive modeling in this paper. The implementation is based on Craig-Bampton method, which is a reduction technique where complex deformable structures are divided into an assemblage of substructures (Ericsson et al 2015). Each substructure has its own mass and stiffness matrix associated with a set of generalized coordinates. The set involves two forms of generalized coordinates, boundary generalized coordinates and internal generalized coordinates. The boundary generalized coordinates prescribe the displacements and rotation at the boundaries while the internal generalized coordinates are related to the free vibration modes of the substructure with completely restrained boundaries. Consequently, the two sets of generalized coordinates become decoupled at the boundaries of the substructure and coupled internally.

\subsection{Contact object}

In this paper, we chose a polygon-based contact solution referring to the previous study result (Elmqvist et al, 
2015). To have a reasonable computation performance, the 2-dimension contact model is built and equipped with Multibody connectors, which can be used seamlessly with rigid multibody system model.

The contact detection is usually composed of broad and narrow detection phase, while in the relay modeling application the latter phase only is good enough. The contact pole surface profile is defined by polygon, as an external object to Modelica. The polygon vertex coordinates array has to be set up by the order of counterclockwise.

Another external object is defined for contact force. The contact model is built by arranging two potential colliding polygons together in pair. Internally, these two bodies have no connector for interaction. Instead, an algorithm is used to monitor polygon objects' relative positions and detect the collision. When the collision occurs, contact force object will be used to compute the contact forces. Externally, this polygon pair has two multibody connectors working as interface to interact with outside multibody system.

The mechanical parts of the relay system are modeled by multibody system at 3D space while the contact model works at 2D space. The mechanical model with ideal driving force is shown in Figure 3. The body with red color represents the flexible beam, body with blue color is rigid body; The component with icon of two colliding polygons is the $2 \mathrm{D}$ contact model handling the two contact bodies.

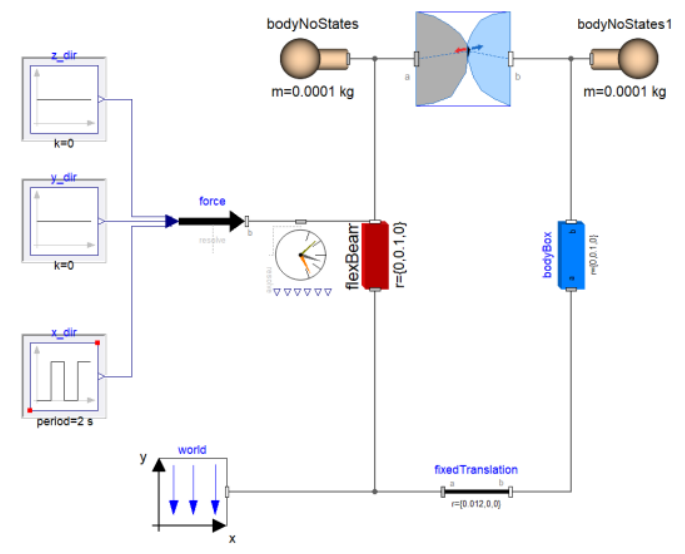

Figure 3. Relay mechanical model using MultiBody and 2D contact model

At the parameter setup diagram of the 2D contact model, the profiles of the contact pole surface can be specified through coordinates array. Table 1 shows the main mechanical parameters of the relay system.

Table 1. Relay mechanical parameters

\begin{tabular}{|l|l|l|}
\hline Parameters & value & unit \\
\hline $\begin{array}{l}\text { Length of the fixed and } \\
\text { moveable beam }\end{array}$ & 100 & $\mathrm{~mm}$ \\
\hline
\end{tabular}

\begin{tabular}{|r|l|l|}
\hline $\begin{array}{l}\text { Dist. between the fixed } \\
\text { \& moveable beams }\end{array}$ & 12 & $\mathrm{~mm}$ \\
\hline Pos. of driving force & 100 & $\mathrm{~mm}$ \\
\hline Flexible beam & & \\
\hline Density & 8.8 & $\mathrm{~g} / \mathrm{cm}^{3}$ \\
\hline Young's modulus & $109 \times 10^{9}$ & $\mathrm{~Pa}$ \\
\hline Shear modulus & $109 \times 10^{9} / 2.6$ & $\mathrm{~Pa}$ \\
\hline Poisson's ratio & 0.3 & 1 \\
\hline Contact poles & & \\
\hline Young's modulus & $200 \times 10^{9}$ & $\mathrm{~Pa}$ \\
\hline Poisson's ratio & 0.3 & 1 \\
\hline Radius of the pole & 5 & $\mathrm{~mm}$ \\
\hline
\end{tabular}

\subsection{Contact normal force}

The contact force is calculated in term of the intersection status between two collision bodies. The force value is volume dependent as in Eq. (2) for polyhedron (Elmqvist et al 2015)

$$
F=E k \sqrt{V d}
$$

where the $E$ is the Young's modulus of the objects, $V$ is the volume of the overlapping region of polyhedron (here, the area of overlapping between polygons), $d$ is the penetration depth and $\mathrm{k}$ is calculated in Eq. (3),

$$
k=\frac{4}{3 \sqrt{\pi}}
$$

This is a generalization of the Hertz model. The force is applied at the centroid of the overlapping region.

\subsection{Contact friction force}

The contact surface profile in this study is of spherical shape. The moveable part is flexible beam, the vibration from the beam will make the contact bounce phenomenon even more complicated since the contact point location may change at each time. To address the slip during tangential contact, the contact model needs to be able to calculate the friction force during the impact process.

A common practice to integrate friction force into multibody system is to approximate the law of friction using a continuous function (Popov, 2010). A tangential velocity dependent function will be used to model the friction force. The function is defined as

$$
F_{f}=\left(\left(2 \mu_{s *}-\mu_{k}\right) \frac{x^{2}}{x^{4}+1}+\mu_{k}-\frac{\mu_{k}}{x^{2}+1}\right) F_{n}
$$




$$
\begin{gathered}
\mu_{s *}=\mu_{s}\left(1-0.09\left(\frac{\mu_{k}}{\mu_{s}}\right)^{4}\right) \\
x=\frac{v_{t}}{v_{s}}
\end{gathered}
$$

where the coefficients $\mu_{s}$ and $\mu_{k}$ are the static and kinetic friction coefficient respectively, $F_{n}$ is the contact normal force, $v_{t}$ is the tangential velocity and $v_{S}$ is the transition velocity from static to kinetic friction. Figure 4 shows the friction as a function of tangential velocity (Elmqvist et al 2015).

Keeping a purely kinetic friction function means that there will never be a completely "stuck" mode. A small tangential force that would not influence a system with Coulomb friction at rest. This friction function will give rise to a tangential speed until a force equilibrium is met.

The transition velocity $v_{s}$ decides how quickly the friction force increases. A smaller value will approximate Coulomb friction better.

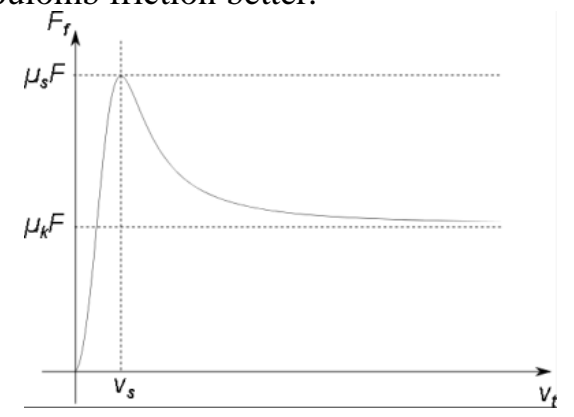

Figure 4. Friction as function of tangential velocity

\subsection{Electromagnetic circuit}

The electromagnetic part is modeled by an equivalent circuit using flux tubes and air-gaps network. Saturation of the magnetic material and dynamical effects can be introduced to the model.

In the current study, the model from Magnetic Library of MSL is used in Figure 5.

The interaction between the subsystems is twofold: firstly, the electromagnetic forces and torques applied on the mechanical subsystem produce deformations and accelerations; Secondly, the motion affects the electromagnetic subsystem by induced electromotive forces and by variations of reluctance.

In this paper, we focus on the former one, that is on the dynamics of the mechanical system driven by force from electromagnetic subsystem. The thrust $F$ developed by a translational electro-magnetomechanical actuator is calculated by following Eq. (7) in the lumped magnetic network models.

$$
F=\frac{1}{2} \sum_{i=1}^{n_{\text {linear }}} V_{m i}^{2} \frac{d G_{m i}}{d x}
$$

where nlinear is the number of flux tube elements with constant relative permeability that change its permeance $G_{m i}$ with armature position (index i), $V_{m i}$ the magnetic voltage across each respective flux tube and $d G_{m i} / d x$ the derivative of the respective permeances with respect to armature position.

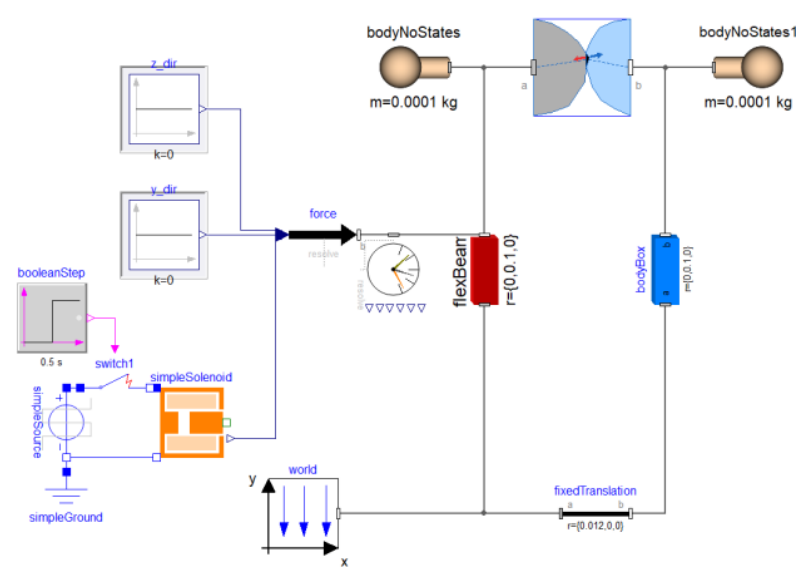

Figure 5. Electromagnetic relay model

The armature rotational dynamic is not considered. Instead a translational solenoid model in MSL.Magnetic with minor customization is used to generate the corresponding thrust force.

\section{Simulation}

\subsection{Contact bouncing suppression}

Eliminating the contact bouncing is of interest in relay design. In Figure 6, the upper plots are the moveable contact position in horizontal direction; the lower plots are the Boolean signal for contact.

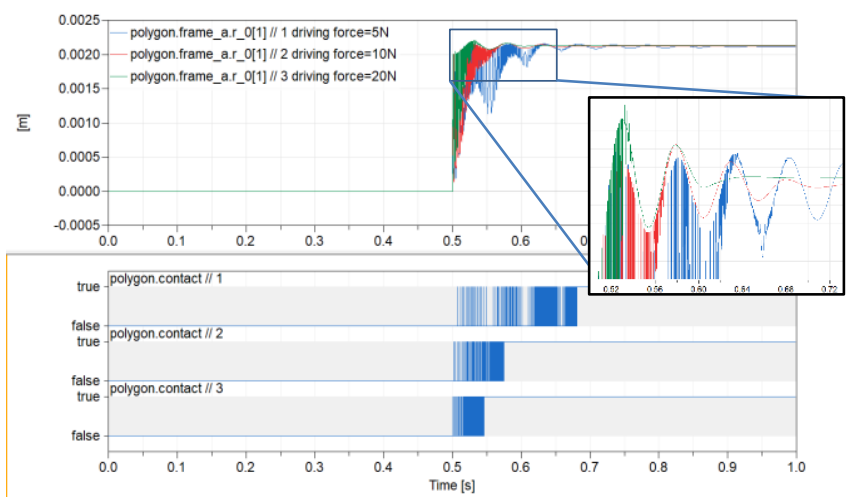

Figure 6. Contact point position horizontal variation (upper); Contact Boolean signal (lower)

The simulation results show that when the driving force applied has different amplitude as $5 \mathrm{~N}, 10 \mathrm{~N}, 15 \mathrm{~N}$, the higher the driving force, the less contact bounce in contact make. This result is consistent with the result in previous studies [McBride W. 1992].

However, when increasing the driving force to $30 \mathrm{~N}$, it is observed that the tangential speed will grow quickly and the friction force correspondingly (Figure 
7). This can lead to the unstable contact that should be avoided.

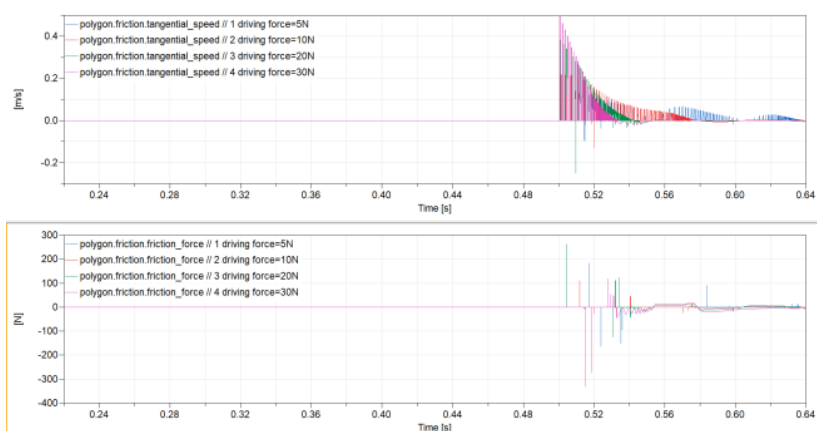

(a) Tangential speed and friction force of the contact under different electromagnetic driving forces

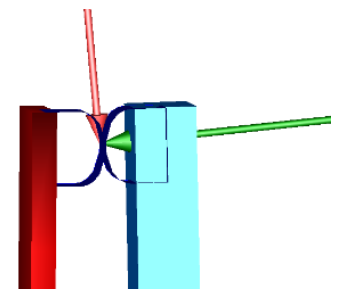

(b) Normal force and friction forces in contact

Figure 7. Speed dependent friction force and normal force

\subsection{Flexible beam vibration}

To identify the effect of moveable beam to the relay contact bounce, the pulse signal for electromagnetic force is set up as period of pulse is $2 \mathrm{~s}$, width of pulse is $50 \%$, start time $=0.15 \mathrm{~s}$ and the amplitude is $5 \mathrm{~N}$. Then the simulation result of the moveable contact pole position in horizontal direction is shown in Figure 8. Continuous vibration is observed after the first contact release in $1.15 \mathrm{~s}$ and the second contact make is under the mixed beam vibration and contact bounce. This result is regarded as a new observation through relay system simulation that has not been reported at the early simulation research.

In the conventional relay system, there is a return spring and the moveable beam is rigid. The relay structure discussed in this paper has no return spring which makes the structure simple and has high structure reliability.

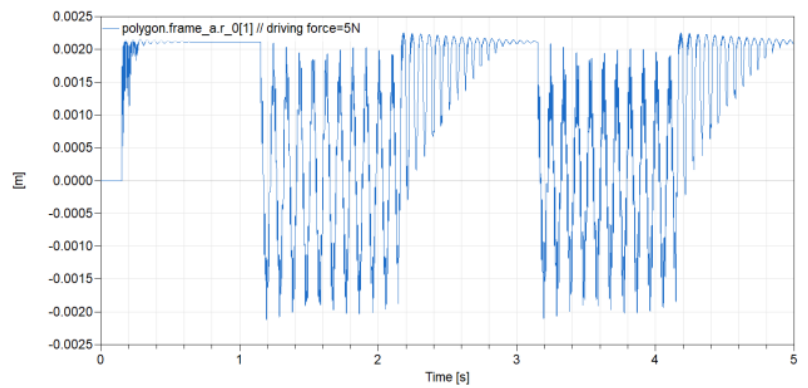

Figure 8. Vibration of the successive contact make
However, to achieve the high robustness in contact make is still a design problem through which to optimize the parameters of the relay system.

In the current simulation study, we simple increase the electromagnetic driving force from $5 \mathrm{~N}$ to $20 \mathrm{~N}$, Figure 9 shows that it is effective to suppress the contact bounce as well, though the number of the contact bounce after the first time will be bigger.
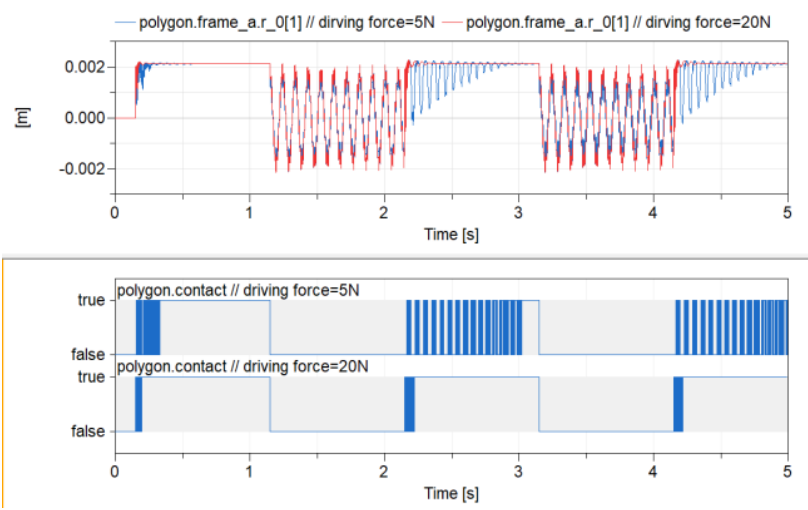

Figure 9. Contact bounce suppression by increasing driving force amplitude from $5 \mathrm{~N}$ to $20 \mathrm{~N}$

\subsection{Contact surface profile change}

In practical, on a worn electrical contact the radius is expected to be dominated by the local roughness rather than the initial radius of the contacts.

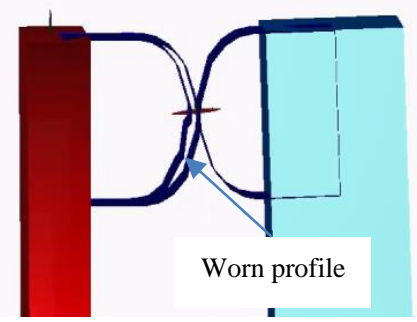

Figure 10. An example of contact pole worn surface profile

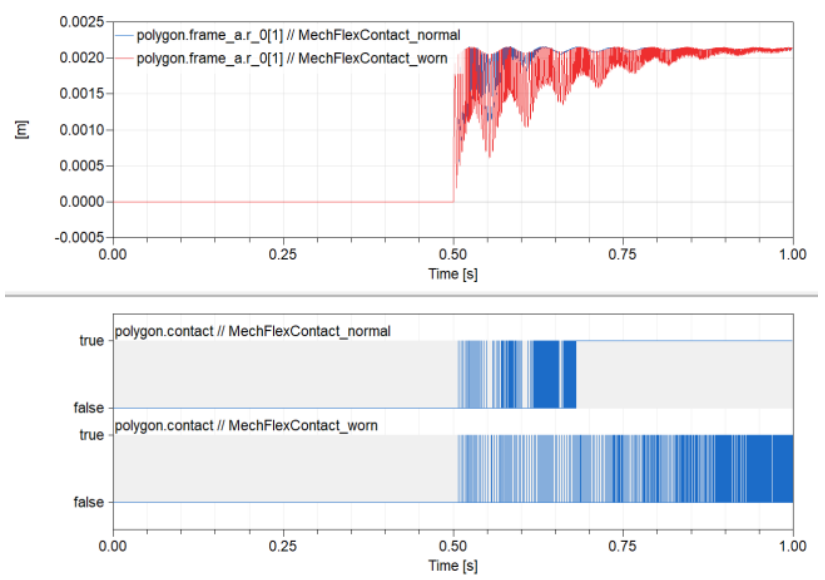

Figure 11. Comparison of the contact bounce between normal profile and worn profile 
It is noticed that the contact bounce with a worn contact pole profile in Figure 10 will be continued longer than a normal case as shown in Figure 11.

Though the mechanism of electric erosion to the contact surface and its effect to the contact dynamics need further research, the model built in the paper is expected to provide simulation support to the study.

\subsection{Electromagnetic response}

For the completeness, the ideal driving force is generated from electromagnetic circuit. The typical responses of the electromagnetic system are shown in Figure 12.
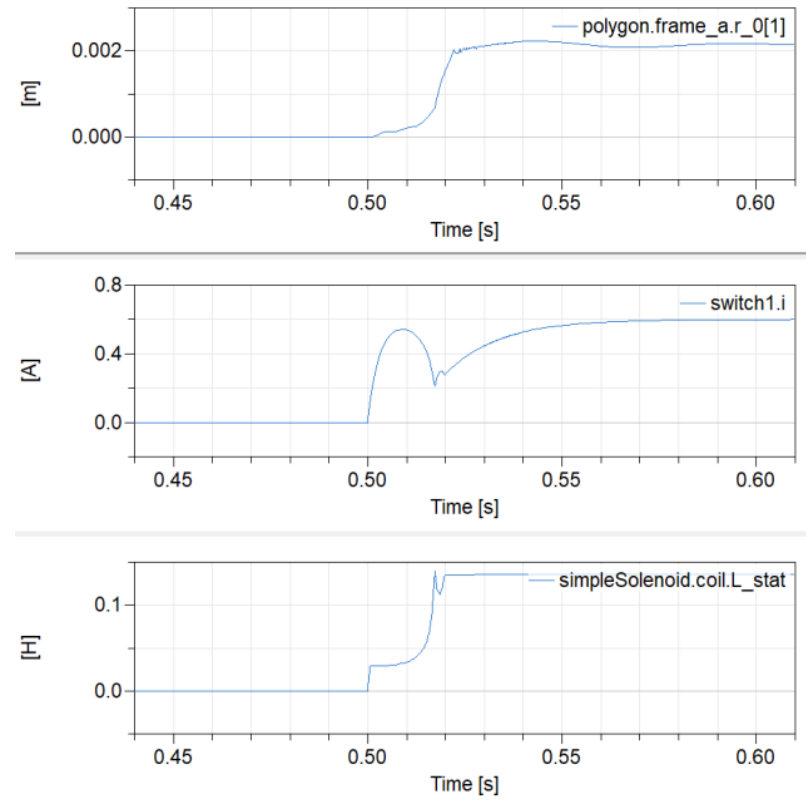

Figure 12. Moveable contact position(upper); Electrical switch current (middle); Magnetic circuit static inductance (lower)

\subsection{Simulation performance}

Using the created model including flexible beam, 3D multibody and 2D contact model, it takes less than 8s to simulate a use case in Figure 9. i.e. 3 contact make process within 5s (Figure 13).

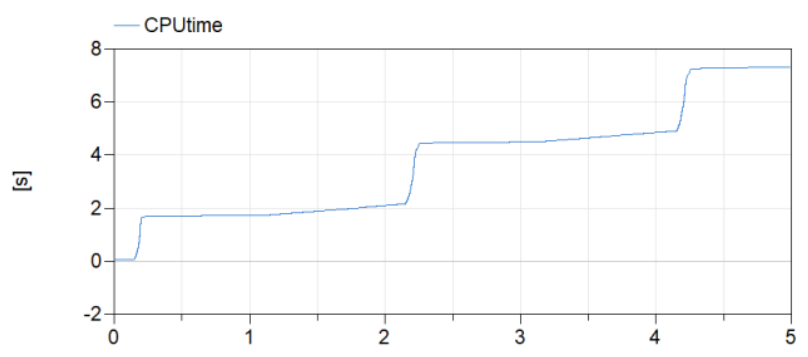

Figure 12. CPU time and event for a $5 \mathrm{~s}$ simulation

\section{Conclusions}

A multi-physics electromagnetic relay model is created where the mechanical part is under multibody framework and electromagnetic part is by equivalent circuit. The main effort is to use the flexible beam represent the relay moveable part and 2D contact model to capture the impact between the moveable and stationary poles. Simulation results show the built model reproduces the physical behavior of the relay, especially the contact bounce phenomenon. Furthermore, the model is easily extended to a simulation for a worn contact surface case, which is expected to help improve the relay design by simulation analysis.

The simulation performance of the created model shows at the similar level with rigid multibody system even though the flexible beam and contact elements are included.

For the future work, the model validation using practical measurement to improve the prediction accuracy is an important step towards practical engineering application. Applying the latest 3D modeling technique is another topic of interest to investigate efficient modeling approach with performance comparison.

\section{Acknowledgements}

The polygon-based collision function is supported by Oskar Åström and Hilding Elmqvist based on the earlier work (Elmqvist et al, 2015).

\section{References}

A. Heckmann, M. Otter, S. Dietz and J. D. López. The DLR FlexibleBody library to model largemotions of beams and of flexible bodies exported from finite element programs. In:5th International Modelica Conference. Wien, 2006, pp. $85-95$.

A. Ericsson, A. Kjellander Efficient Modeling of a Flexible Beam in Dymola using Coupled Substructures in A Floating Frame of Reference Formulation, Master Thesis, Department of Mechanical Engineering, Lund University, 2015

A. Neumayr, M. Otter. Component-Based 3D Modeling of Dynamic Systems Proceedings of the $1^{\text {st }}$ American Modelica Conference, pp. 175-186, Oct. 2018, Cambridge, Massachusetts, USA. doi:10.3384/ ECP18154175

A Neumayr, Martin O. Algorithms for Component-Based 3D Modeling Proc. of the $13^{\text {th }}$ International Modelica Conference, pp. 383-392, Mar. 2019, Regensburg, Germany. doi:10.3384/ ECP19157383

F. Oestersötebier, P. Wang, A. Trächtler A Modelica Contact Library for Idealized Simulation of Independently Defined Contact Surfaces, Proceedings of the 10th International Modelica Conference, pp929-937, March 10-12, 2014, Lund, Sweden, doi: 10.3384/ECP14096929

FlexBody Library, https://www.claytex.com/products/ 
Gianni F. Bruno S. Andrea R. Multibody Model of a Motorbike with a Flexible Swingarm Proc. of the 10th International Modelica Conference, pp. 273-282, March 10-12, 2014, Lund, Sweden, doi: 10.3384/ECP14096273

H. Elmqvist, A. Goteman, V. Roxling, and T. Ghandriz. Generic Modelica Framework for MultiBody Contacts and Discrete Element Method. In Peter Fritzson and Hilding Elmqvist, editors, Proc. of the 11th International Modelica Conference. LiU Electronic Press, Sept. 2015. URL http://www.ep.liu.se/ ecp/118/046/ecp15118427.pdf.

H. Nouri., N. Larsen, T. S. Davies. Contact Bounce simulation using MATLAB. IEEE Xplore, 9(2):284-288, November 1997. doi: 10.1109/HOLM.1997.638054

John W. McBride, Suleiman M. Sharkh. Electrical Contact Phenomena During Impact, IEEE Transactions on Component, Hybrids, and Manufacturing Technology. Vol., 15, No. 2, April 1992.

M. Otter, H. Elmqvist, and J. Diaz Lopez. Collision Handling for the Modelica MultiBody Library. In Gerhard Schmitz, editor, Proc. of the 4th International Modelica Conference, March 2005. https://modelica.org/events/Conference2005/ online_proceedings/Session1/Session1a4.pdf.

S. Kondo, Y. Yokote, H. Tanaka Simulation Technology to Predict Dynamic Motion of Relay (In Japanese), OMRON Technics. Vol. 51.021JP, 2019.4.

Valentin L. Popov. Contact Mechanics and Friction, Physical Principles and Applications, Springer. e-ISBN 978-3-64210803-7. doi:10.1007/978-3-642-10803-7.

Xiong J., He J. Zang C. Dynamic Analysis of Contract of Aerospace Relay Based on Finite Difference Method. Chinese Journal of Aeronautics, 22 (2009):262-267. doi:10.1016/S10000-9361(08)60097-7. 